\title{
THE URCA PROCESS IN CONVECTIVE CORES*
}

\author{
(Abstract)
}

S. TSURUTA

NASA, Goddard Space Flight Center, Greenbelt, Md., U.S.A.**

and

A. G. W. CAMERON

Harvard College Observatory, Cambridge, Mass., U.S.A.

The importance of the URCA neutrino process in convective cores in late stages of stellar evolution was already pointed out in this Symposium. In pre-supernova stages, Paczyński (1972) regarded convective motion as a stellar vibration. We explored the possibility for a more realistic approach. As a first step, we assumed convective motion as a fully developed turbulence, which is represented by superposition of eddies of different velocities and sizes obeying the Kolmogoroff spectrum. Using the Monte Carlo method, we followed the path of a lump of matter, with emission of neutrinos (and antineutrinos) whenever appropriate (though electron captures inside and through beta decays outside the URCA shell), until a steady state is reached. The calculations were carried out for a typical pre-supernova model (similar to that of Paczyński).

The results are: (a) when the stellar luminosity $L \simeq 100 L_{\odot}$, the URCA neutrino luminosity $L_{v}^{u}$ is $\sim 10^{38} \mathrm{erg} \mathrm{s}^{-1}$, somewhat less than but comparable with Paczyński's result, while (b) a significant increase in $L_{v}^{u}$ is noted for $L \simeq 10^{6} L_{\odot}$. In both (a) and (b), the convective velocity is sufficiently fast, so that the corresponding Reynolds number is far greater than the critical value. Therefore, the assumption of fully developed turbulence should be perfectly valid. It may be noted that the random walk approach we adopted for the direction of motion is not valid in the presence of discontinuity in composition. However, already at $L \bumpeq 100 L_{\odot}$, the timescale for beta processes $\left(\sim 10^{5} \mathrm{~s}\right)$ is longer than the convective timescale $\left(\sim 10^{4} \mathrm{~s}\right)$, and mixing of elements is nearly complete. In view of the absence of better convective theories in degenerate cores, our results may prove to be useful.

\section{Reference}

Paczyński, B.: 1972, Astrophys. Letters 11, 53.

* This paper was presented by S. Tsuruta.

** Present address, Astronomy Centre, University of Sussex, Falmer, Brighton, U.K. 